The Astrophysical Journal, 665:000-000, 2007 August 20

(C) 2007. The American Astronomical Society. All rights reserved. Printed in U.S.A.

\title{
ELECTRON FLUX SPECTRAL IMAGING OF SOLAR FLARES THROUGH REGULARIZED ANALYSIS OF HARD X-RAY SOURCE VISIBILITIES
}

\author{
Michele Piana, ${ }^{1}$ Anna Maria Massone, ${ }^{2}$ G. J. Hurford, ${ }^{3}$ Marco Prato, ${ }^{4}$ A. Gordon Emslie, ${ }^{5}$ \\ Eduard P. Kontar, ${ }^{6}$ and Richard A. Schwartz ${ }^{7}$ \\ Received 2007 March 16; accepted 2007 May 3
}

\begin{abstract}
We introduce a new method for imaging spectroscopy analysis of hard X-ray emission during solar flares. The method avoids the "traditional" noise-sensitive step of stacking independent images made in different count-based energy intervals. Rather, it involves regularized inversion of the count visibility spectra (i.e., the two-dimensional spatial Fourier transforms of the spectral image) to obtain smoothed (regularized) forms of the corresponding electron visibility spectra. Application of conventional visibility-based imaging algorithms then yields images of the electron flux that vary smoothly with energy. We apply the method to a solar flare observed on 2002 February 20 by the RHESSI instrument. The event is characterized by two bright footpoints with a more diffuse emission between them. Analysis of the regularized electron flux images reveals that the electron flux spectra at the footpoints are systematically harder than those in the region between the footpoints and that the observed degree of hardening is consistent with that produced by Coulomb collisions between an acceleration site high in the corona and the dense chromospheric footpoint regions.
\end{abstract}

Subject headings: methods: data analysis — Sun: flares — Sun: X-rays, gamma rays techniques: image processing — techniques: spectroscopic

\section{INTRODUCTION}

The Reuven Ramaty High-Energy Solar Spectroscopic Imager (RHESSI) produces hard X-ray and $\gamma$-ray images with the finest angular and spectral resolutions ever achieved (Lin et al. 2002); imaging spectroscopy analysis of this data is a powerful tool with which to explore the underlying physics of particle acceleration and transport in solar flares. Traditional imaging spectroscopy methods (e.g., Emslie et al. 2003) start by constructing twodimensional maps of the source at different count energies by applying image processing algorithms (e.g., back projection, CLEAN, Maximum Entropy, Pixon). This results in a series of images which are consistent both with the broad assumptions of the particular algorithm used and with the imaging information contained in the data. Spatially resolved count spectra are then obtained from this set of images by selecting particular regions in the field of view and comparing the intensity in those regions as a function of count energy. Finally, the corresponding spatially resolved electron spectra are constructed by applying regularized spectral inversion methods (e.g., Brown et al. 2006) to the spatially resolved count spectra.

In this paper, we introduce a new approach to imaging spectroscopy which is optimized to the distinctive way in which spatial information is encoded in the RHESSI data. The RHESSI

\footnotetext{
${ }^{1}$ Dipartimento di Informatica, Università di Verona, Ca' Vignal 2, Strada le Grazie 15, I-37134 Verona, Italy; michele.piana@univr.it.

2 CNR-INFM LAMIA, via Dodecaneso 33, I-16145 Genova, Italy; massone@ ge.infm.it.

${ }^{3}$ Space Sciences Laboratory, University of California, Berkeley, 8 Gauss Way, Berkeley, CA 94720-7450; ghurford@ssl.berkeley.edu.

${ }^{4}$ Dipartimento di Matematica, Università di Modena e Reggio Emilia, via Campi 213/b, I-41100 Modena, Italy; marco.prato@unimo.it.

5 Department of Physics, Oklahoma State University, Stillwater, OK 74078; gordon.emslie@okstate.edu.

6 Department of Physics and Astronomy, University of Glasgow, Glasgow G12 8QQ, Scotland, UK; eduard@astro.gla.ac.uk.

${ }^{7}$ CUA (Catholic University) and LSSP at NASA Goddard Space Flight Center, code 671.1, Greenbelt, MD 20771; richard.schwartz@gsfc.nasa.gov.
}

instrument employs a rotation modulation collimator (RMC) imaging technique, in which rapid time variations of the detected counts are affected by the placement of a set of RMCs, each with a different pitch, in front of each detector. Spatial information is encoded in the temporal modulation of the detected flux (Hurford et al. 2002). As the RMC rotates, the amplitude and phase of this pseudoperiodic modulation over a limited range of angles provides a direct, calibrated measurement of a single Fourier component of the source distribution. Such a Fourier component is termed a visibility (Prince et al. 1988) and is the same quantity provided by the correlated signal from a pair of antennas in a radio interferometer. In this case, the spatial frequency of the measured visibility is determined by the angular resolution of the RMC and its instantaneous orientation. Combining data from multiple RMCs at a variety of orientations, the set of visibilities can then be used to reconstruct the spatial distribution of the source. Since visibilities can be summed linearly, this perspective on the data provides a convenient basis for combining data from multiple rotations into a tractable number of visibility measurements with well-defined statistical errors.

The "traditional" approach to imaging spectroscopy, in which images at different count energies are "stacked" and compared, not only fails to take full advantage of the particular nature in which spatial information is contained in the RHESSI data, but also has two significant drawbacks:

1. While imaging algorithms can reduce statistical and pointresponse artifacts in each image, they are completely ineffective in smoothing along the count-energy direction, so that recovered images corresponding to adjacent energy bins can exhibit substantial differences;

2. Owing to these energy-dependent fluctuations, the determination of the count spectrum at a particular point $(x, y)$ (or, more accurately, a particular region $[x \pm \Delta x, y \pm \Delta y]$ ) in the source image can be problematic, as is the determination of the statistical error on the count flux. As is well known (e.g., Craig $\&$ Brown 1986), such noise-related spectral variations are greatly 
magnified upon performing a spectral inversion to obtain the corresponding electron spectrum.

In addition, as with any indirect imaging technique, the incomplete spatial frequency sampling results in spatial sidelobes in the point-response function which can cause contamination by neighboring sources. Further, the statistical noise from all source components contributes to the noise in each selected region of the source.

It is crucial to recognize that it is not the observed counts (or even photons) that are of interest per se, but rather the electrons that produce them: the real science goal is to obtain physically plausible (i.e., "sufficiently smooth") electron spectra throughout the source. Our new method of imaging spectroscopy analysis therefore involves an interchange of two steps in the data processing chain. First, one applies a count to electron inversion algorithm to obtain smoothed electron spectra at each point in the spatial frequency domain. Once such electron flux visibility spectra have been obtained, they can be processed using standard image reconstruction techniques to yield electron flux images for the entire field of view. Since the electron flux visibility spectra are regularized, so also are the corresponding electron flux spectra at each location in the image. This renders these spatially resolved spectra more suitable for further analysis.

We perform the count to electron inversion step using the familiar Tikhonov (1963) regularization technique that has proven so effective (e.g., Piana et al. 2003) in the inference of spatially integrated electron spectra $\bar{F}(E)$ from observations of spatially integrated count (or photon) spectra $I(\epsilon)$. Applied to visibilities, the Tikhonov regularization method forces smoothness in the inferred electron visibility spectra at each point in the spatial frequency domain and thus enhances real features that persist over a relatively wide energy band, while suppressing noise-related features that show up only over a narrow range of energies. The combination of visibility data and Tikhonov regularization methodology therefore allows us to derive the most robust information on the spatial structure of the electron flux spectrum image, the key quantity of physical interest.

In $\S 2$ we present a formal development of the necessary quantities and their interrelationships. In $\S 3$ we apply the technique to a flare observed on 2002 February 20 and demonstrate the ensuing enhancements in the quality of the results obtained. In $\S 4$ we discuss the results obtained and their physical significance. We summarize in $\S 5$.

\section{METHODOLOGY}

The definitions of key quantities of interest, and the relationships among them, are developed formally in the Appendix. Here we simply underscore the essence of the method.

The observed photon spectrum image $I(x, y ; \epsilon)\left(\right.$ photons $\mathrm{cm}^{-2}$ $\mathrm{s}^{-1} \mathrm{keV}^{-1} \operatorname{arcsec}^{-2}$ ) produced by a region of a hard X-ray source is a threefold convolution of the line-of-sight column density $N(x, y)$ (particles per $\mathrm{cm}^{2}$ of projected source area), the electron flux spectrum $\bar{F}(x, y, E)$ (electrons $\mathrm{cm}^{-2} \mathrm{~s}^{-1} \mathrm{keV}^{-1}$ ) averaged along the line of sight, and the photon production cross section $Q(\epsilon, E)\left(\mathrm{cm}^{2} \mathrm{keV}^{-1}\right)$. Assuming that the instrument response can be expressed through a linear detector response matrix (see footnote 11 in the Appendix for a discussion of this point), it follows that the observed count spectrum image $J(x, y ; q)\left(\right.$ counts $\mathrm{cm}^{-2}$ $\left.\mathrm{s}^{-1} \mathrm{keV}^{-1} \operatorname{arcsec}^{-2}\right)$ is linearly related to the quantity $N(x, y) \bar{F}(x, y$; $E) K(q, E)$. Here $K(q, E)$ is the differential count cross section $\left(\mathrm{cm}^{2} \mathrm{keV}^{-1}\right)$, representing the cross section for production of a count of energy $q$ by an electron of energy $E$, which takes into account both the detector response to modulated photons and the blanket and attenuator transmission effects.

Since the spatial Fourier transform is an inherently linear operation, the fundamental linearity of the relationship between count and electron spectra is preserved when transforming from the spatial domain to the visibility (spatial frequency) domain. Thus the count visibility spectrum $V(u, v ; q)\left(\right.$ counts $\mathrm{cm}^{-2} \mathrm{~s}^{-1}$ $\left.\mathrm{keV}^{-1}\right)$, defined as the two-dimensional Fourier transform $\mathcal{F}^{2}$ of the observed image $J(x, y ; q)$ at count energy $q(\mathrm{keV})$,

$$
\begin{aligned}
V(u, v ; q) & =\mathcal{F}^{2}(J(x, y ; q)) \\
& \equiv \int_{x} \int_{y} J(x, y ; q) e^{2 \pi i(u x+v y)} d x d y
\end{aligned}
$$

is linearly related to the electron flux visibility spectrum,

$$
W(u, v ; E)=a^{2} \mathcal{F}^{2}(N(x, y) \bar{F}(x, y ; E))
$$

(electrons $\mathrm{cm}^{-2} \mathrm{~s}^{-1} \mathrm{keV}^{-1}$ ), where the unit conversion factor $a=7.25 \times 10^{7} \mathrm{~cm} \mathrm{arcsec}^{-1}$ at $R=1$ AU. Indeed, as shown formally in the Appendix, the relationship between $V(u, v ; q)$ and $W(u, v ; E)$ is

$$
V(u, v ; q)=\frac{1}{4 \pi R^{2}} \int_{q}^{\infty} W(u, v ; E) K(q, E) d E .
$$

Equation (3) is formally identical to the relation between the spatially integrated photon spectrum and the source-integrated electron spectrum, viz. (Brown et al. 2003),

$$
I(\epsilon)=\frac{1}{4 \pi R^{2}} \int_{\epsilon}^{\infty} \bar{n} V \bar{F}(E) Q(\epsilon, E) d E,
$$

and so can be solved for the visibilities $W(u, v ; E)$ from the observed count visibility spectra $V(u, v ; q)$ by applying the Tikhonov regularization technique that has proven so effective (e.g., Piana et al. 2003) in the solution of equation (4) for $\bar{n} V \bar{F}(E)$ given $I(\epsilon)$.

To briefly summarize the Tikhonov methodology, equation (3) is first discretized in both count and electron energy spaces to yield, at each sampled point $(u, v)$ in the spatial frequency domain, the data visibility vector $\boldsymbol{V}_{[u, v]}$ (the elements of which de- Q2 pend on count energy $q$ ) and the source visibility vector $\boldsymbol{W}_{[u, v]}$ (the elements of which depend on electron energy $E$ ). These are related through the matrix equation

$$
\boldsymbol{V}_{[u, v]}=\boldsymbol{K} \cdot \boldsymbol{W}_{[u, v]},
$$

where $\boldsymbol{K}$ is the kernel matrix, the elements of which are formed from the values of $K(q, E)$ at the discretized count and electron energy points. Then the zero-order regularization problem,

$$
\left\|\boldsymbol{V}_{[u, v]}-\boldsymbol{K} \cdot \boldsymbol{W}_{[u, v]}\right\|^{2}+\lambda_{[u, v]}\left\|\boldsymbol{W}_{[u, v]}\right\|^{2}=\text { minimum, }
$$

is solved for $\boldsymbol{W}_{[u, v]}$ given the prescribed visibility vector $\boldsymbol{V}_{[u, v]}$ at each sampled point in $(u, v)$ space, using an appropriate value (see below) of the regularization parameter $\lambda_{[u, v]}$. This results in electron visibility spectra that are "smooth" in the sense that the large variations in $W(u, v ; E)$ from energy bin to energy bin are suppressed. This technique therefore enhances spatial features (Fourier components) that persist over a wide range of energies and suppresses (noise) features that exist over only a limited subset of energy bins. Once the electron visibility spectra have been 

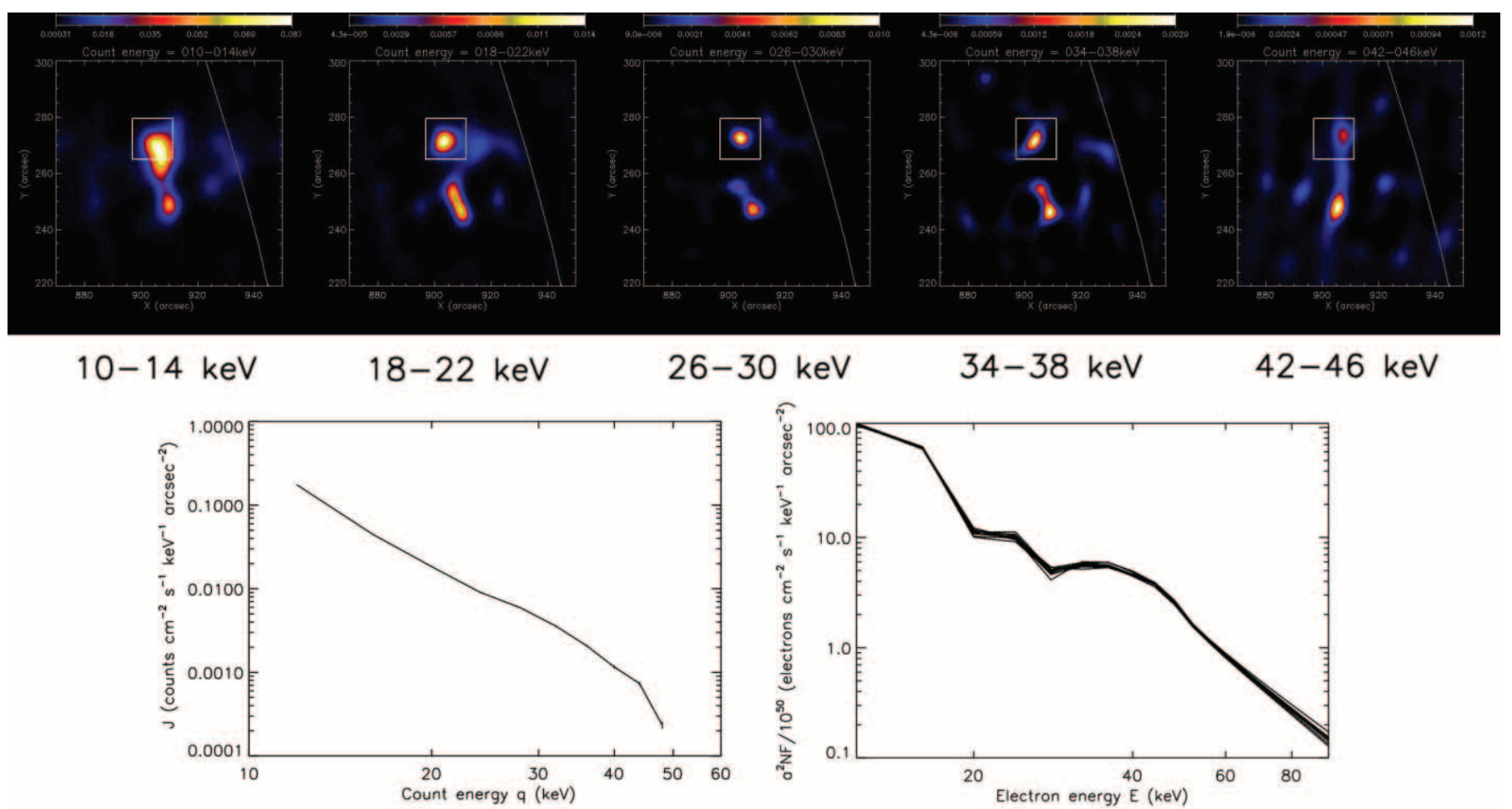

FIG. 1.-Top panels: Count images for the 2002 February 20 (11:06:02-11:06:34 UT) event for the energy intervals shown, produced using the MEM-NJIT algorithm (Bong et al. 2006). Bottom panels: Areally averaged count spectrum (left panel; counts $\mathrm{cm}^{-2} \mathrm{~s}^{-1} \mathrm{keV}^{-1} \operatorname{arcsec}^{-2}$ ) and electron flux confidence strip spectra (right panel; electrons $\mathrm{cm}^{-2} \mathrm{~s}^{-1} \mathrm{keV}^{-1} \operatorname{arcsec}^{-2}$ ) for the footpoint region highlighted in the images.

determined, the electron flux spectral image may be determined through the inverse Fourier transform of equation (2), namely

$N(x, y) \bar{F}(x, y ; E)=\frac{1}{a^{2}} \int_{u} \int_{v} W(u, v ; E) e^{-2 \pi i(u x+v y)} d u d v$.

\section{APPLICATION TO DATA}

We illustrate the method by applying it to data obtained near the peak of the C7.5 flare of 2002 February 20 (11:06:0211:06:34 UT), using visibilities from RHESSI RMCs 3 through 9, corresponding to spatial resolutions from $\sim 7^{\prime \prime}$ to $\sim 183^{\prime \prime}$. For comparison purposes, we first construct count images by means of the visibility technique and apply the "traditional" imaging spectroscopy approach, in which count images in different energy bands are compared. After discussing the drawbacks of this traditional method, we use our new method to obtain more physically useful electron flux maps of the flare.

The traditional method begins by converting the X-ray count rate data to a set of visibilities. This requires preselecting the number of angular intervals (roll bins) per rotation. The number of roll bins should be maximized to avoid degradation of sensitivity near the edge of the field of view. However, for this application, each roll bin must contain at least one complete modulation cycle to enable the visibility to be well measured. Using an iterative technique, we maximized the number of roll bins for each detector subject to this constraint and then used a $\chi^{2}$ analysis to determine statistically acceptable visibilities. Then, since $V(u, v)$ and $V(-u,-v)$ are complex conjugates (see eq. [1]), the visibilities measured at angles separated by $180^{\circ}$ are combined to improve the signal-to-noise ratio. Finally, the error bars on the real and imaginary parts of each visibility for each energy channel are computed by propagating the statistical error in the counts through to the calculation of each visibility. The resulting visibilities are used as input to the Maximum Entropy (MEM-NJIT) algorithm (Bong et al. 2006), as implemented in the Solar SoftWare (SSW) package, to produce $\left(80^{\prime \prime} \times 80^{\prime \prime}\right)$ maps with $0.4^{\prime \prime}$ pixels. This was done for $164 \mathrm{keV}$ wide energy intervals from 10 to $74 \mathrm{keV}$.

Figure 1 shows some of these count-based images. Two bright features, which we interpret as emission from chromospheric footpoints, are apparent. In addition, there is some evidence for a "strand" of emission linking the two bright features; this we interpret as emission from the coronal region of the magnetic loop linking the footpoints. The lower left panel of Figure 1 shows the areally averaged count spectrum (counts $\mathrm{cm}^{-2} \mathrm{~s}^{-1} \mathrm{keV}^{-1}$ $\operatorname{arcsec}^{-2}$ ) for the northern footpoint region highlighted by the square in each image; this spectrum has been constructed by averaging, for each energy channel, the intensities of the pixels that constitute the highlighted region. (To get the total count spectrum for the region [counts $\mathrm{cm}^{-2} \mathrm{~s}^{-1} \mathrm{keV}^{-1}$ ], simply multiply by the area of the region, in this case $14.4 \times 14.4=207.36 \operatorname{arcsec}^{2}$.) The error bars have been computed as the combination of a count (Poisson) error plus background noise. The lower right panel shows the recovered electron flux spectrum confidence strip (i.e., a series of realizations of the electron flux spectrum, each based on a different noisy realization of the data; see Piana 1994) for this feature. Each electron spectrum realization was obtained by inverting the count spectrum using the SSW zero-order regularization method applied by Piana et al. (2003) for spatially integrated spectroscopy.

The count spectrum is conspicuously weak in the $26-30 \mathrm{keV}$ image. This leads to a relatively flat count spectrum in this range (lower left panel of Fig. 1) and hence (since the electron spectrum is, crudely, related to the derivative of the photon spectrum; Brown 1971) to a dip in the recovered electron spectrum (lower 

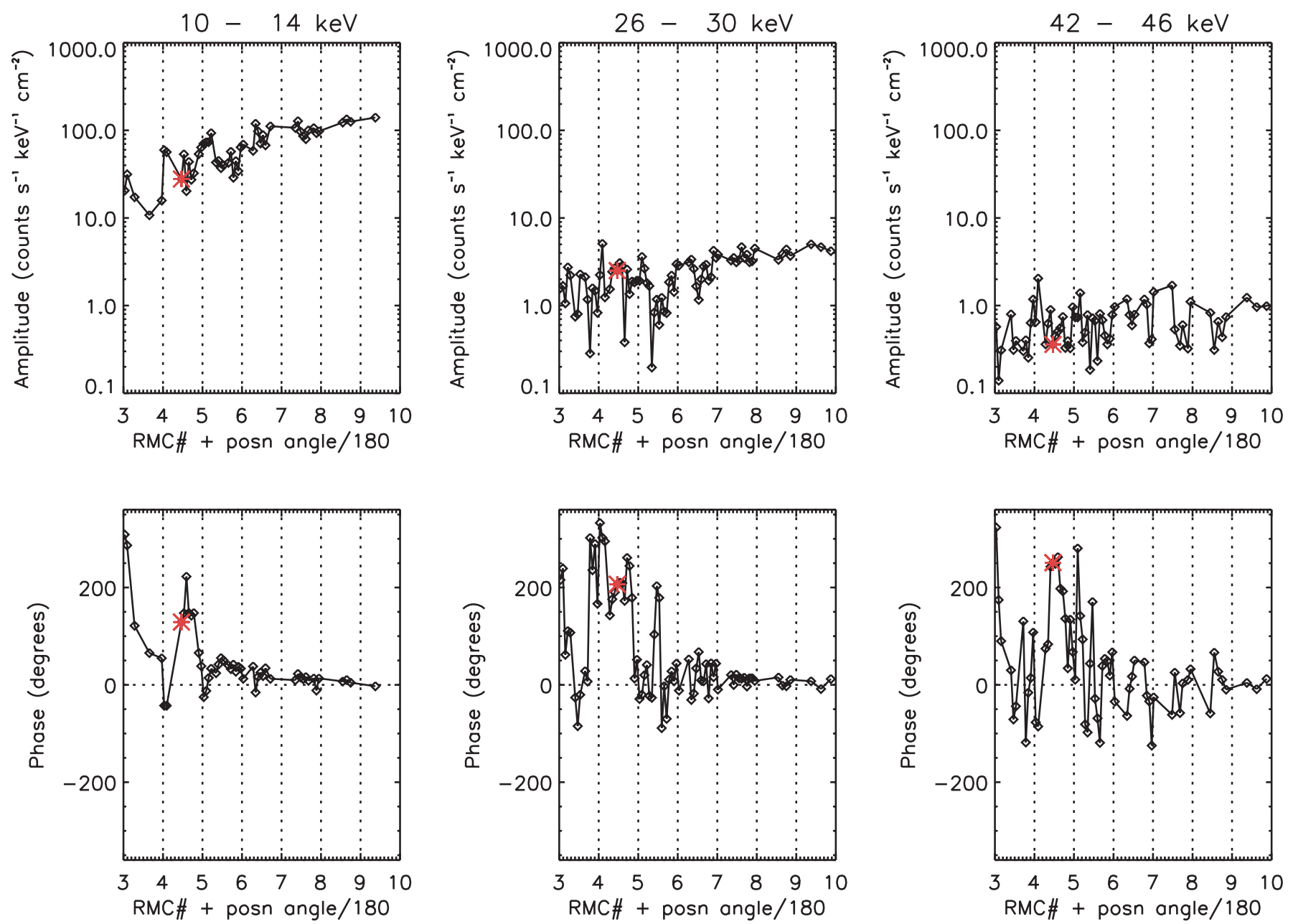

FIG. 2.- Observed count visibilities (amplitude and phase) for three representative energy bands. In each panel, the region between each pair of dotted vertical lines

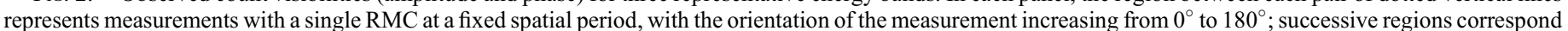

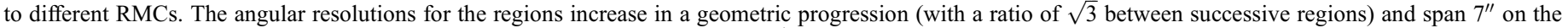

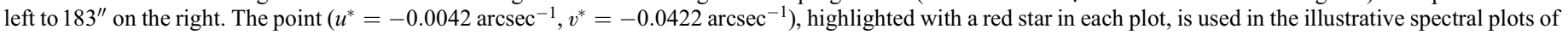
Fig. 3.

right panel) for this feature. Although such spectral dips have been inferred for spatially integrated electron spectra (e.g., Piana et al. 2003), the spatially integrated count spectra on which such spatially integrated electron spectra are based are not subject to the imaging artifacts that render suspect the count spectra determined for a particular spatial region. Therefore it is possible, or indeed likely, that the feature in Figure 1 is not real, but rather an artifact imposed by isolating attention on a limited range of spatial coordinates, rather than on the overall patterns (Fourier components) of emission present in the spatially integrated emission.

As discussed in $\oint 1$, because spatial information is fundamentally encoded by RHESSI in Fourier components, rather than in "pixels," a more cogent approach to imaging spectroscopy involves performing the count to electron inversion step in the spatial frequency domain, i.e., on the visibility data. By focusing on the information in distinct Fourier components, we remove the deleterious effects of imaging artifacts that are evident in the more "traditional" approach to imaging spectroscopy.

Figure 2 shows the amplitude (upper panels) and phase (lower panels) of the count visibilities $V(u, v ; q)$ (counts $\mathrm{cm}^{-2} \mathrm{~s}^{-1} \mathrm{keV}^{-1}$ ) for the same event and time interval as Figure 1, for three count energy ranges. The amplitude of the visibilities generally in- creases with increasing grid pitch [decreasing value of the corresponding spatial frequency $\left.\left(u^{2}+v^{2}\right)^{1 / 2}\right]$.

Highlighted by a red star in each plot in Figure 2 is the (somewhat arbitrary) point $\left(u^{*}=-0.0042 \operatorname{arcsec}^{-1}, v^{*}=-0.0422 \operatorname{arcsec}^{-1}\right)$; this point corresponds to a spatial periodicity $1 /\left(u^{* 2}+v^{* 2}\right)^{1 / 2}=$ 23.6", which is the spatial periodicity corresponding to (i.e., twice the angular resolution of ) RHESSI grid 4. The top panels of Figure 3 show the amplitude $\left|V\left(u^{*}, v^{*} ; q\right)\right|$ and phase $\operatorname{Arg}\left(V\left(u^{*}, v^{*} ; q\right)\right)$ of the differential count visibility spectrum for this representative point in the spatial frequency domain.

In order to preserve the inherent linearity of the process, a polar to rectangular transformation was performed to convert the amplitude and phase information into real and imaginary components $\operatorname{Re}\left(V\left(u^{*}, v^{*} ; q\right)\right)$ and $\operatorname{Im}\left(V\left(u^{*}, v^{*} ; q\right)\right)$. Each of these components was then subjected to the regularized inversion analysis of equation (6) to obtain the real and imaginary parts of the corresponding (regularized) electron visibility spectrum $W\left(u^{*}, v^{*} ; E\right)$ at the point $\left(u^{*}, v^{*}\right)$. Through an inverse rectangular to polar transform, we then recover the amplitude and phase of the electron visibility spectrum $W\left(u^{*}, v^{*}, E\right)$ at this particular point, as shown in the bottom panels of Figure 3 .

Repeating this regularized inversion process for each sampled point in the $(u, v)$ plane (using a value of the regularization parameter 

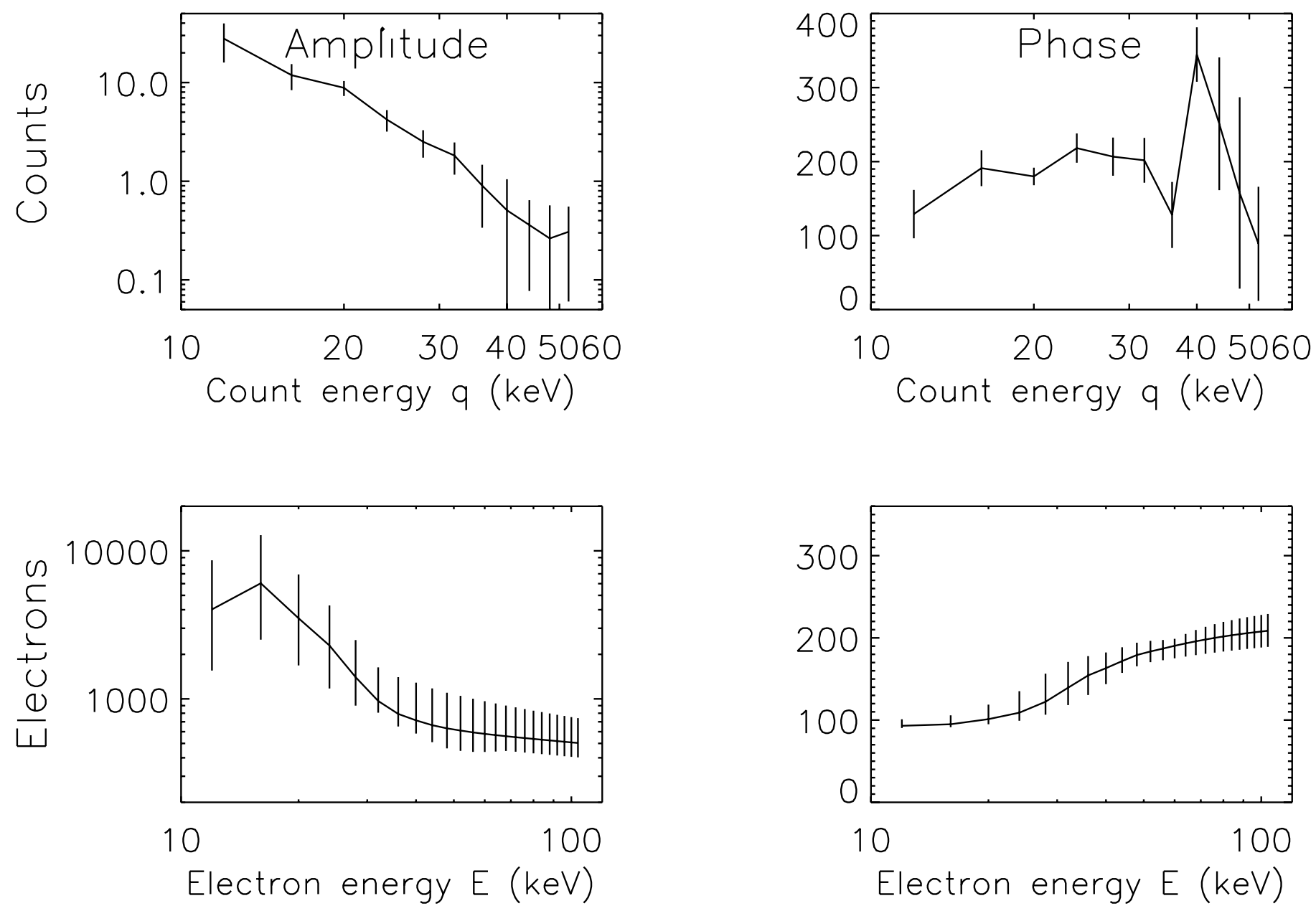

FIG. 3.-Top panels: Amplitude (left panel; counts $\mathrm{cm}^{-2} \mathrm{~s}^{-1} \mathrm{keV}^{-1}$ ) and phase (right panel; degrees) of the count visibility spectrum $V\left(u^{*}, v^{*} ; q\right)$ at the point $\left(u^{*}=-0.0042, v^{*}=-0.0422\right)$ in the spatial frequency domain. Bottom panels: Amplitude (left panel; in units of $\left.10^{50} \mathrm{electrons} \mathrm{cm}^{-2} \mathrm{~s}^{-1} \mathrm{keV}^{-1}\right)$ and phase $($ right panel; degrees) of the corresponding electron spectrum visibilities $W\left(u^{*}, v^{*} ; E\right)$ at the same $(u, v)$ point, obtained through regularized inversion of eq. (3) using the zeroorder Tikhonov method.

$\lambda_{[u, v]}$ appropriate $^{8}$ to each sampled $(u, v)$ point), we arrive at complete information on the electron flux visibility spectrum. This information is presented in Figure 4 in the same format as Figure 2.

We can now use the set of electron flux visibility spectra to construct electron spectral flux images in each energy range. Images of the electron flux spectrum $\bar{F}(x, y ; E)$, recovered by applying the MEM-NJIT algorithm (Bong et al. 2006), are shown in Figure 5. These images represent the quantity of key physical interest.

Figure 5, like Figure 1, shows evidence for two footpoints, again connected by a "strand" of coronal flux. To the extent that variations in count intensity are a consequence of data noise, the regularization algorithm used to develop the electron flux images of Figure 5 removes such irregularities, resulting in a more coherent variation of source structure with energy. Consequently, the electron flux images vary much more smoothly with energy, and the coronal "strand" is more persistent at low energies.

\footnotetext{
${ }^{8}$ The value of the regularization parameter $\lambda_{\left[u^{*}, v^{*}\right]}$ was chosen using the " $3 \sigma$ cumulative residual criterion" approach discussed in detail by Piana et al. (2003) and Kontar et al. (2004); in general, such a procedure for determining $\lambda_{[u, v]}$ results in more faithful representations of electron flux spectra than the commonly used "discrepancy principle."
}

The footpoints in the electron images are seen to persist up to electron energies of $\sim 75 \mathrm{keV}$, an energy significantly greater than the maximum photon energy used. As pointed out by Kontar et al. (2004), information on the electron spectrum at high energies is indeed contained in the photon spectrum at lower energies and can be faithfully extracted using the Tikhonov regularization procedure.

It is instructive to reconstruct the count images corresponding to the regularized electron spectral flux images in Figure 5 and compare them with the original spatial images obtained through processing of the raw count visibility data using the MEM algorithm. This comparison is presented in Figure 6. The top row of figures shows the recovered count images at the energies shown, while the bottom row reproduces the original count-based images from Figure 1.

The original count-based images (lower row of images in Fig. 6) show evidence principally of a double-footpoint structure, with some additional evidence for an extension of the emission into the region between the footpoints (see, e.g., the $18-22 \mathrm{keV}$ and $42-46 \mathrm{keV}$ images). However, there is no clear systematic variation with count energy $q$, either of the intensity of this "strand" emission or of the relative intensity of the two footpoints. By contrast, the count images deduced from the regularized electron flux images (upper row of images in Fig. 6) show much more clearly the evolution of the spatial structure with energy. The "strand" of emission between the footpoints is clearly evident 

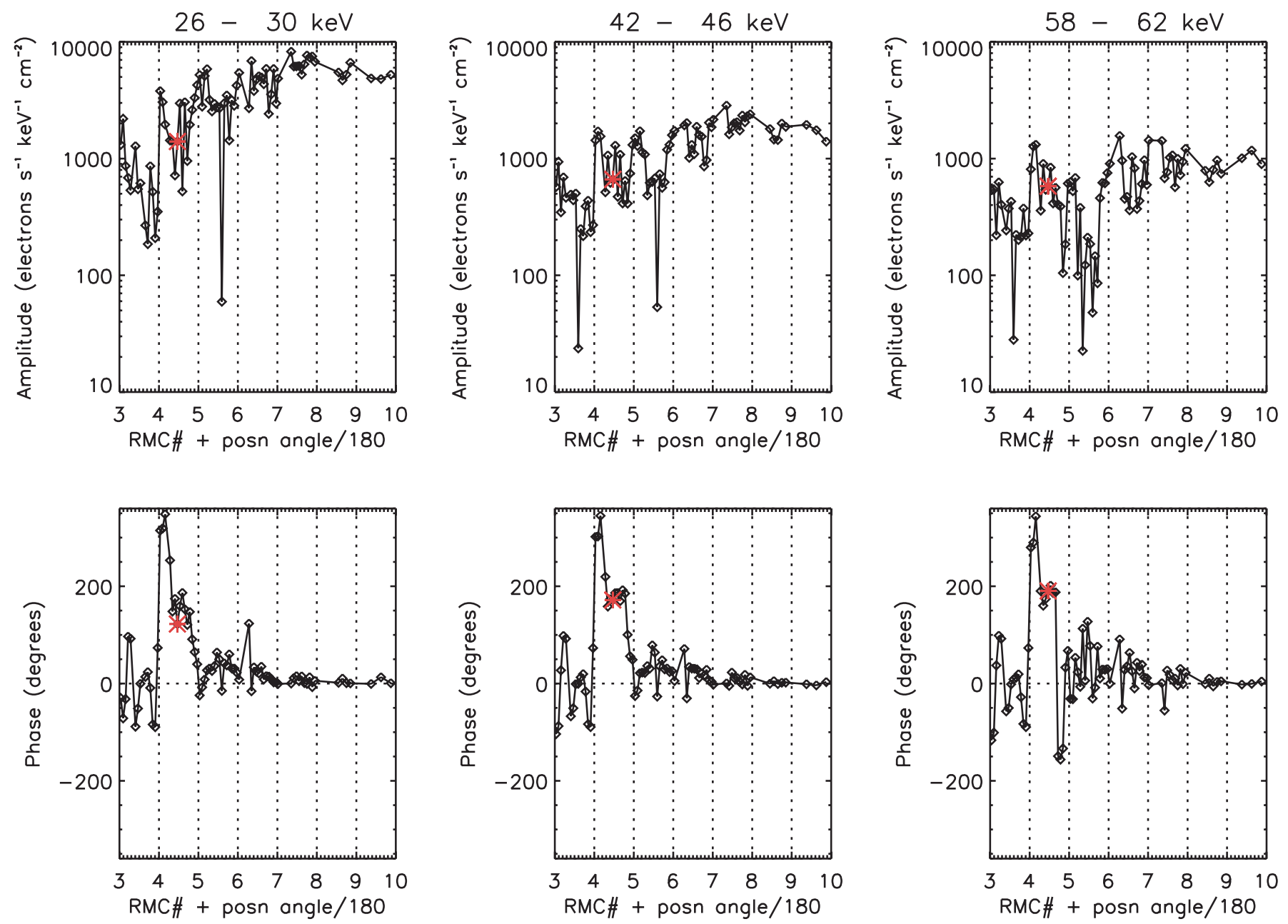

Fig. 4.- Recovered regularized electron flux visibilities (scaled by $10^{-50}$ ), presented as in Fig. 2 . The sharp minima in the amplitude plots are real and reflect the twocomponent nature of the source. The $y$-intercept to the right is determined by the total flux, while the rate at which the amplitudes fall off (to the left) is determined by the size of the sources and reflects, for example, the larger source size at $26-30 \mathrm{keV}$. The broad similarity of the phase plots reflects the broadly similar location of the sources at various electron energies.

up to $30 \mathrm{keV}$, but diminishes rapidly at higher energies, and the relative intensity of the two footpoint sources is more independent of count energy $q$. These physically plausible enhancements in the image structure are recovered through use of the visibilitybased regularized inversion technique, because of its inherent requirement that the source structure vary smoothly from one electron energy $E$ to the next. This requirement in turn forces the count images to change more smoothly with count energy $q$ than do the images deduced directly from the (noisy) data.

\section{PHYSICAL IMPLICATIONS OF THE RESULTS}

The electron spectral flux images of Figure 5 are quite plausibly interpreted in terms of the collisional thick target model (Brown 1971) of hard X-ray emission in solar flares.

Consider three different spatial subregions in the source, labeled in Figure 7. Two of these regions correspond to the footpoint sources visible at higher energies, and the other one to similarly sized regions located approximately midway between the two footpoints. The lower panel of Figure 7 shows the areally averaged ${ }^{9}$ electron flux spectra (electrons $\mathrm{cm}^{-2} \mathrm{~s}^{-1} \mathrm{keV}^{-1} \operatorname{arcsec}^{-2}$ )

\footnotetext{
9 To get the total count spectrum for each region (counts $\mathrm{cm}^{-2} \mathrm{~s}^{-1} \mathrm{keV}^{-1}$ ), simply multiply the areally averaged spectrum by the area of that region, viz. $14.4 \times 14.4=207.36 \operatorname{arcsec}^{2}$ (Footpoint 1$), 22.8 \times 9=205.2 \operatorname{arcsec}^{2}$ (middle), and $14.4 \times 14.4=207.36 \operatorname{arcsec}^{2}$ (Footpoint 2).
}

for each of these three subregions. The region labeled "Footpoint 1" is identical to the region highlighted in Figure 1; comparison of the electron flux spectra for this region (Figs. 1 and 7) show that the "dip" at $\sim(26-30) \mathrm{keV}$ obtained using the "traditional" approach to imaging spectroscopy is indeed an artifact of the data truncation and overspill issues associated with identification of the flux in a local spatial region; the real electron flux spectrum in this region is smooth and monotonically decreasing with energy $E$.

At low energies $E \lesssim 60 \mathrm{keV}$, the electron flux at the more southern footpoint (Footpoint 2) is much smaller than that at the more northern footpoint (Footpoint 1). However, the spectrum of Footpoint 2 is very hard $(\delta \simeq 1)$, and by $\sim 60 \mathrm{keV}$, the electron flux at each footpoints has become roughly equal, as is apparent from the spatial images.

Above $E \sim 40 \mathrm{keV}$, the spectra corresponding to the two footpoint regions are visibly flatter (harder) than that corresponding to the region between these footpoints. Such a result is qualitatively consistent with the acceleration of electrons in a source midway between the footpoints and the subsequent propagation of these electrons to the footpoints. To concentrate the observed degree of spectral hardening in the footpoints constrains the intervening column density to an upper limit $N<E^{2} / 2 K \sim 2 \times$ $10^{17}[E(\mathrm{keV})]^{2} \sim 3 \times 10^{20} \mathrm{~cm}^{-2}$. (Here $K=2 \pi e^{4} \Lambda \simeq 2.6 \times$ $10^{-18} \mathrm{~cm}^{2} \mathrm{keV}^{2}$, where $e$ is the electronic charge and $\Lambda$ the 

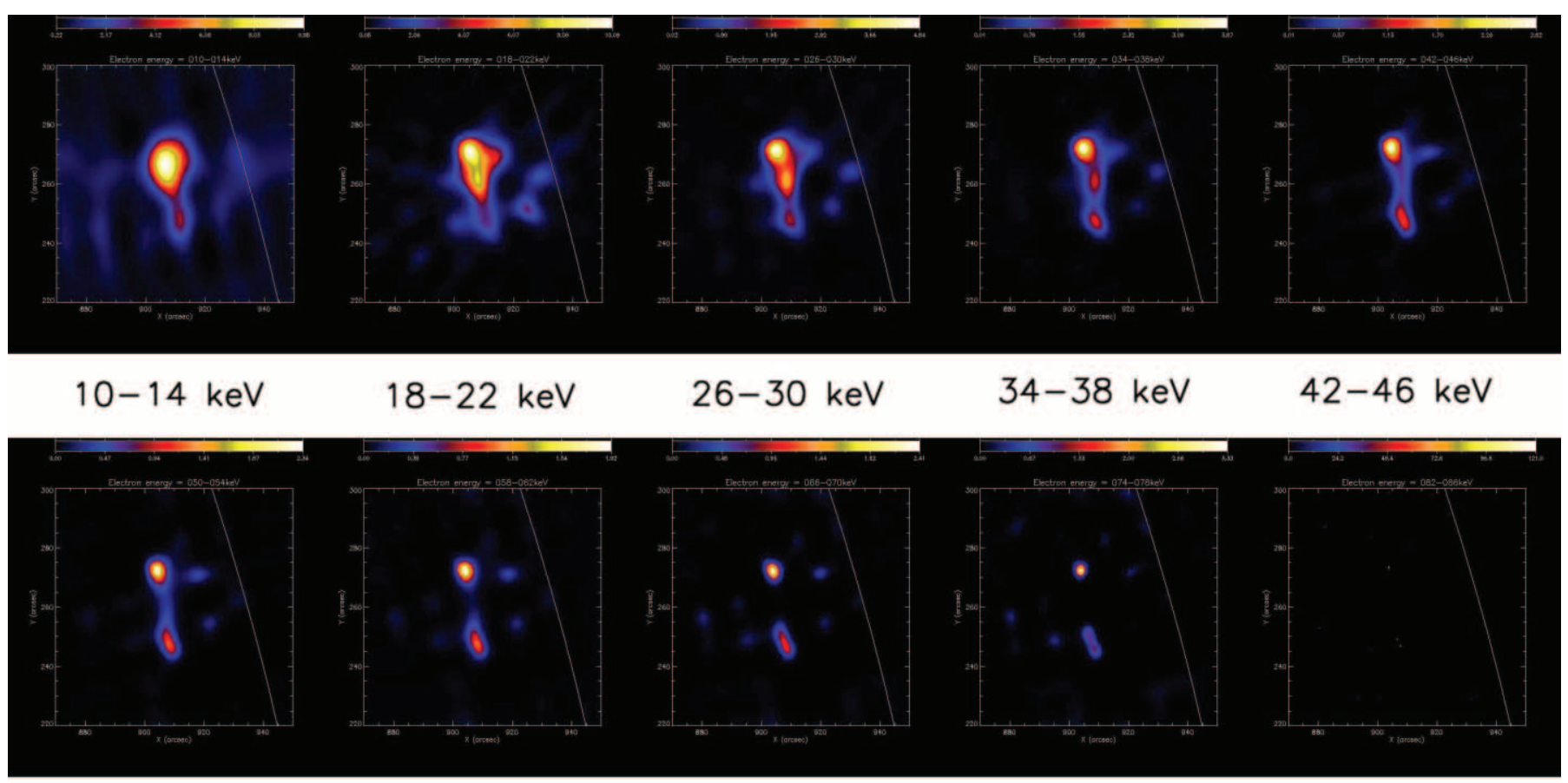

50-54 keV

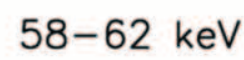

\section{6-70 keV}

\section{4-78 keV}

82-86 keV

FIG. 5.-Electron flux spectral images corresponding to the regularized electron flux spectral visibilities of Fig. 4, obtained through application of the MEM-NJIT algorithm (Bong et al. 2006).

Coulomb logarithm.) This in turn establishes an upper limit on the coronal density $n \sim N / d$, where $d$ is the distance between the coronal source and the footpoint parallel to the guiding magnetic field. The plane-of-sky projected distance between the "middle" and "footpoint" sources is $\sim 10^{\prime \prime} \sim 7 \times 10^{8} \mathrm{~cm}$. Assuming a semicircular geometry for the loop connecting the footpoints, $d \sim(\pi / 2)$ times this projected distance, i.e., $\sim 10^{9} \mathrm{~cm}$. We hence infer that the coronal density $n<3 \times 10^{11} \mathrm{~cm}^{-3}$, an entirely reasonable constraint.

\section{SUMMARY}

We have developed and illustrated the effectiveness of a new approach to solar hard X-ray imaging spectroscopy. In this

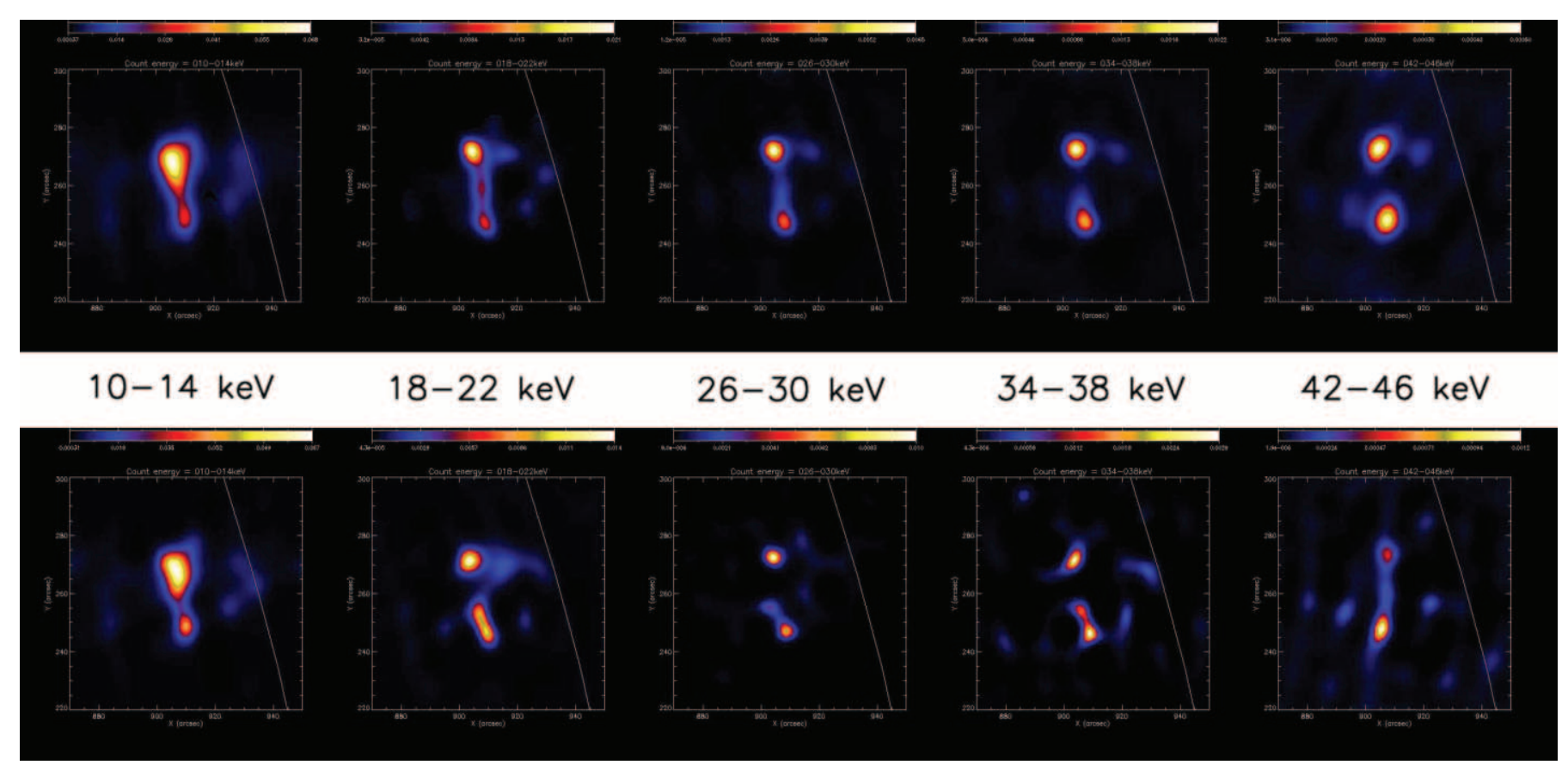

Fig. 6.- Regularized count-based images corresponding to the electron flux spectral images of Fig. 5 (top panels), compared with the original images from Fig. 1 (bottom panels). 

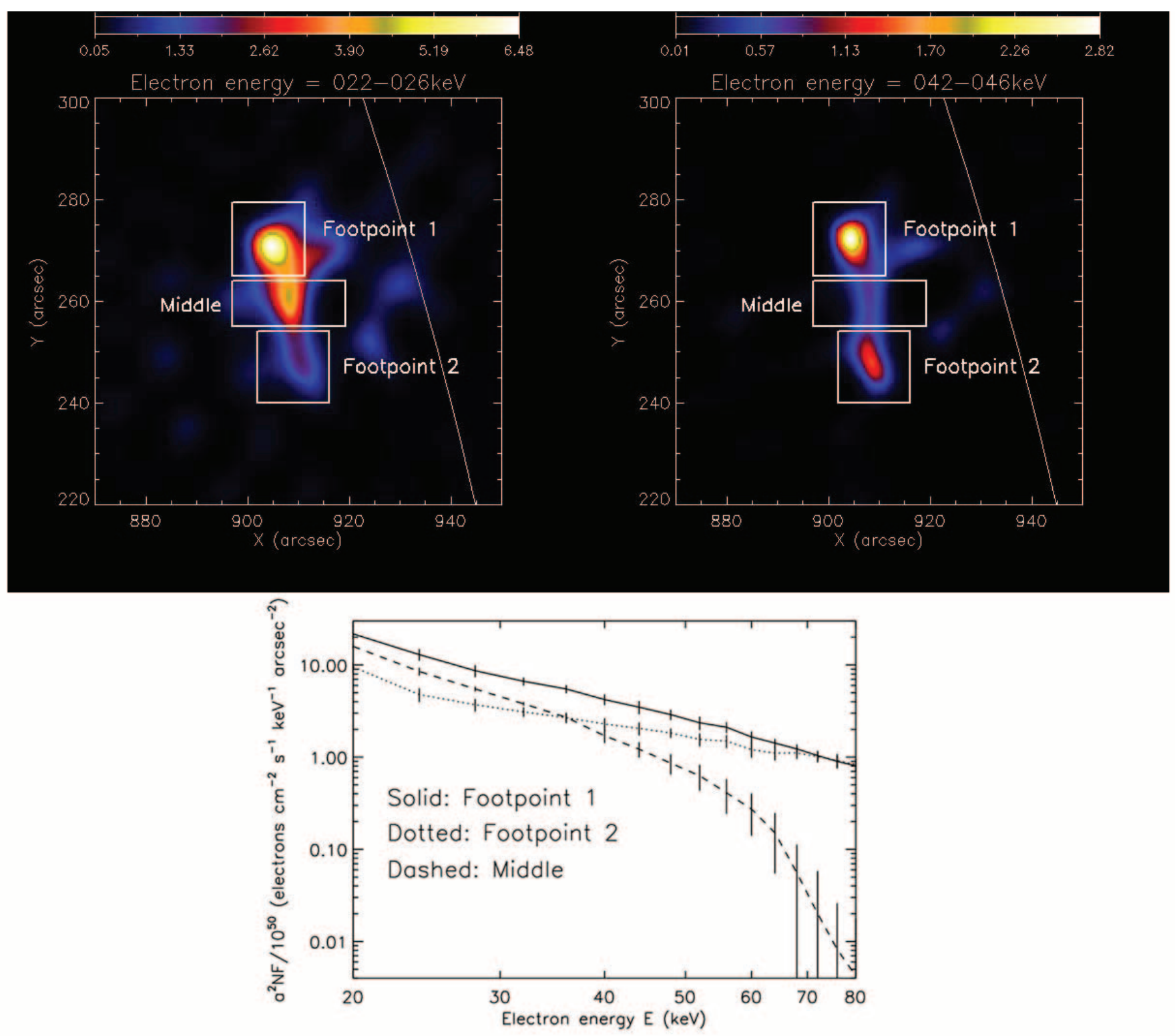

Fig. 7.-Top panels: Electron images in the energy ranges $22-26 \mathrm{keV}(l e f t)$ and $42-46 \mathrm{keV}$ (right). Three subregions of interest are labeled on each image. Two of these correspond to bright footpoint-like sources, and one to a region midway between the footpoints. Bottom panel: Areally averaged electron flux spectra (electrons $\mathrm{cm}^{-2} \mathrm{~s}^{-1}$ $\mathrm{keV}^{-1} \operatorname{arcsec}^{-2}$ ) for each of the three subregions shown.

approach, two-dimensional Fourier transforms of the image in the count domain are transformed, through a regularized inversion procedure that enhances features that persist over a range of energy channels, into Fourier transforms of the electron flux maps. A final image reconstruction based on an inverse Fourier transform then gives the electron flux maps themselves. Because data obtained through rotating modulation collimator instruments, such as RHESSI, are concentrated into a relatively small number of discrete Fourier components ("visibilities"), this approach is highly effective at analyzing such data and results in recovered spectra that are determined more precisely than with a method that involved regularized inversion of the count spectrum within a spatial subregion of the source (which necessarily involves a combination of spatial Fourier components).

Application of the method to a flare on 2002 February 20 yielded a series of electron flux images. Varying smoothly with energy, these images in turn permitted recovery of smooth, regularized, electron flux spectra at different regions in the source.
Such smooth, regularized, electron flux spectra contrast with those obtained using the more "traditional" approach to imaging spectroscopy (e.g., Fig. 1), in which unphysical features may result from focusing on a particular spatial region.

For the illustrative event studied, the electron spectra at the two bright chromospheric footpoints evident in the images were systematically harder than the spectrum obtained at similarly sized region between the footpoints. Such a spectral hardening is broadly consistent with collisional modification of an accelerated electron beam if the intervening density is less than $3 \times 10^{11} \mathrm{~cm}^{-3}$.

In future papers, we intend to apply our new technique to a variety of flare events. The resulting sample of electron flux spectrum images provides the required input to the next stage of inquiry, wherein the nature of the physical processes affecting the bremsstrahlung-producing electrons is determined through analysis (e.g., Emslie et al. 2001) of variation in the electron flux spectrum throughout the source. 
G. J. H. was supported in part by NASA contract NAS598033; A. G. E. by NASA grant NNG04G063G and by subcontract SA4878-26308 from the University of California, Berkeley; E. P. K. by a PPARC Advanced Fellowship; and R. A. S. under
Cooperative Agreement NNG06GB96A with The Catholic University of America. The overall effort has greatly benefited from support by a grant from the International Space Science Institute (ISSI) in Bern, Switzerland.

\section{APPENDIX}

\section{DERIVATION OF THE FUNDAMENTAL RELATION BETWEEN COUNT AND ELECTRON VISIBILITIES}

Define a Cartesian coordinate system $(x, y, z)$ such that $(x, y)$ (in units of arcseconds) represents a location in the image plane and $z(\mathrm{~cm})$ represents distance along the line of sight into the source. Let the local density of target particles along the line-of-sight depth $\ell(x, y)(\mathrm{cm})$ be $n(x, y, z)\left(\mathrm{cm}^{-3}\right)$, and let the differential electron flux spectrum (electrons $\left.\mathrm{cm}^{-2} \mathrm{~s}^{-1} \mathrm{keV}^{-1}\right)$ at the point $(x, y, z)$ in the source be $F(x, y, z ; E)$.

Since the source is optically thin, the relation between $F(x, y, z ; E)$ and the corresponding observed photon spectrum image I( $x, y$; $\epsilon)$ (photons $\mathrm{cm}^{-2} \mathrm{~s}^{-1} \mathrm{keV}^{-1} \operatorname{arcsec}^{-2}$ ) is

$$
I(x, y ; \epsilon)=\frac{a^{2}}{4 \pi R^{2}} \int_{E=\epsilon}^{\infty} \int_{z=0}^{\ell(x, y)} n(x, y, z) F(x, y, z ; E) Q(\epsilon, E) d z d E,
$$

where $a=7.25 \times 10^{7} \mathrm{~cm}_{\operatorname{arcsec}}^{-1}$ at $R=1 \mathrm{AU}$, and $Q(\epsilon, E)\left(\mathrm{cm}^{2} \mathrm{keV}^{-1}\right)$ is the cross section ${ }^{10}$ for emission of a photon at energy $\epsilon$.

We define the mean electron flux spectrum $\bar{F}(x, y ; E)$ (electrons $\mathrm{cm}^{-2} \mathrm{~s}^{-1} \mathrm{keV}^{-1}$ at the Sun) by

$$
\bar{F}(x, y ; E)=\frac{1}{N(x, y)} \int_{z=0}^{\ell(x, y)} n(x, y, z) F(x, y, z ; E) d z,
$$

where the column density $\left(\mathrm{cm}^{-2}\right)$ at each point $(x, y)$ in the image is given by $N(x, y)=\int_{z=0}^{\ell(x, y)} n(x, y, z) d z$. Then, by equations $(\mathrm{A} 1)$ and (A2), we may write

$$
I(x, y ; \epsilon)=\frac{a^{2}}{4 \pi R^{2}} \int_{E=\epsilon}^{\infty} N(x, y) \bar{F}(x, y ; E) Q(\epsilon, E) d E .
$$

Next we introduce spatial frequencies $u$ and $v$ in the $x$ - and $y$-directions, respectively, and define the count visibility spectrum $V(u, v ; q)$ (counts $\mathrm{cm}^{-2} \mathrm{~s}^{-1} \mathrm{keV}^{-1}$ ) as the two-dimensional spatial Fourier transform of the count spectrum image $J(x, y ; q)\left(\mathrm{counts}^{-2} \mathrm{~s}^{-1}\right.$ $\left.\mathrm{keV}^{-1} \operatorname{arcsec}^{-2}\right)$ :

$$
V(u, v ; q)=\int_{x} \int_{y} J(x, y ; q) e^{2 \pi i(u x+v y)} d x d y .
$$

The count spectrum and photon spectrum images are related by the instrument's detector response matrix. Hence we may write

$$
V(u, v ; q) d q=\int_{x} \int_{y} \int_{\epsilon=q}^{\infty} D(q, \epsilon) I(x, y ; \epsilon) e^{2 \pi i(u x+v y)} d \epsilon d x d y,
$$

where the dimensionless quantity $D(q, \epsilon)$ is the differential element of the detector response matrix ${ }^{11}$ corresponding to the generation of a count with energy in the energy range $[q, q+d q]$ from a photon in the energy range $[\epsilon, \epsilon+d \epsilon]$.

Combining equations (A3) and (A5) gives the rather formidable expression

$$
V(u, v ; q) d q=\frac{a^{2}}{4 \pi R^{2}} \int_{x} \int_{y} \int_{\epsilon=q}^{\infty} \int_{E=\epsilon}^{\infty}[N(x, y) \bar{F}(x, y ; E)] D(q, \epsilon) Q(\epsilon, E) e^{2 \pi i(u x+v y)} d E d \epsilon d x d y
$$

which provides the formal relationship between $N(x, y) \bar{F}(x, y ; E)$, the quantity of most direct physical interest, and the observed count visibility spectra $V(u, v ; q)$. We now introduce the count cross section $K(q, E)\left(\mathrm{cm}^{2} \mathrm{keV}^{-1}\right)$ through the expression

$$
K(q, E) d q=\int_{\epsilon=q}^{\infty} D(q, \epsilon) Q(\epsilon, E) d \epsilon
$$

\footnotetext{
10 The form of the quantity $Q(\epsilon, E)$ depends on the emission process being considered. In principle, this could include a host of emission processes, such as gyrosynchrotron emission, inverse Compton emission, free-bound emission, and bremsstrahlung. We here take the form of $Q(\epsilon, E)$ as that corresponding to bremsstrahlung, and we use the isotropic form of the cross section given by Koch \& Motz (1959).

${ }^{11}$ The range of $\epsilon$ corresponding to a count of energy $q$ is taken to be $[q, \infty)$; only photons of energy $\geq q$ can generate a count of energy $q$. This (linear) formalism therefore ignores the possibility of the creation of a count of energy $q$ from the arrival at the same detector of two (or more) photons of energy $<q$ within a very short time interval. This "pulse pileup" process is intrinsically nonlinear (the detector response matrix depends on the incoming photon flux) and so cannot be readily accommodated within the present formalism. Our analysis will therefore be restricted to medium-flux events for which pileup is not likely to be significant.
} 
and the electron flux visibility spectrum (electrons $\mathrm{cm}^{-2} \mathrm{~s}^{-1} \mathrm{keV}^{-1}$ )

$$
W(u, v ; E)=a^{2} \int_{x} \int_{y} N(x, y) \bar{F}(x, y ; E) e^{2 \pi i(u x+v y)} d x d y .
$$

With these definitions, and reversing the order of integration with respect to $\epsilon$ and $E$, equation (A6) can be written as the straightforward integral equation

$$
V(u, v ; q)=\frac{1}{4 \pi R^{2}} \int_{q}^{\infty} W(u, v ; E) K(q, E) d E
$$

that appears in equation (3) of the text.

Bong, S. C., Lee, J., Gary, D. E., \& Yun, H. S. 2006, ApJ, 636, 1159 Brown, J. C. 1971, Sol. Phys., 18, 489

Brown, J. C., Emslie, A. G., Holman, G. D., Johns-Krull, C. M., Kontar, E. P., Lin, R. B., Massone, M. A., \& Piana, M. 2006, ApJ, 643, 523

Brown, J. C., Emslie, A. G., \& Kontar, E. P. 2003, ApJ, 595, L115

Craig, I. J. D., \& Brown, J. C. 1986, Inverse Problems in Astrophysics (London: Adam Hilger)

Emslie, A. G., Barrett, R. K., \& Brown, J. C. 2001, ApJ, 557, 921

Emslie, A. G., Kontar, E. P., Krucker, S., \& Lin, R. P. 2003, ApJ, 595, L107

Hurford, G. J., et al. 2002, Sol. Phys., 210, 61

\section{REFERENCES}

Koch, H. W., \& Motz, J. W. 1959, Rev. Mod. Phys., 31, 920

Kontar, E. P., Piana, M., Massone, A. M., Emslie, A. G., \& Brown, J. C. 2004 , Sol. Phys., 225, 293

Lin, R. P., et al. 2002, Sol. Phys., 210, 3

Piana, M. 1994, A\&A, 288, 949

Piana, M., Massone, A. M., Kontar, E. P., Emslie, A. G., Brown, J. C., \& Schwartz, R. A. 2003, ApJ, 595, L127

Prince, T. A., Hurford, G. J., Hudson, H. S., \& Crannell, C. J. 1988, Sol. Phys., 118,269

Tikhonov, A. N. 1963, Soviet Math. Dokl., 4, 1035 


\section{AUTHOR QUERIES}

Q1 Au: Your article has been edited for grammar, consistency, and to conform to journal style (see Instructions to Authors). To expedite publication, we generally do not query every routine grammatical and style change made to a manuscript, although all substantive changes have been noted. Please review the article carefully. Note that we may be unable to make changes that conflict with journal style, obscure meaning, or create grammatical or other problems. Also note that article proofs via PDF do not show corrections that will have been made by the typesetter's proofreader, which generally include corrections of errors of page layout, figure placement, and spacing and font mistakes; feel free to mark any errors you notice in these areas. When sending us your corrections, if you are writing them by hand, please print clearly. If sending a fax, please do not write too close to the margins of the page, as these are often cut off in fax transmission. Finally, please note that a delayed, incomplete, or illegible response may delay the publication of your article while we contact you.

Q2 Au: ApJ uses bold italic symbols for vectors and bold symbols for vector operators. Please check that these have been changed correctly throughout your paper. 